\title{
Barriers to an Effective Maternal Health Service Delivery Network: A Qualitative Study among Health Providers in Legazpi City, Albay
}

\author{
Ma. Stephanie Fay S. Cagayan, ${ }^{1}$ Rita Mae Ang-Bon, ${ }^{2}$ Fernando B. Garcia, Jr., ${ }^{3}$ Filomena S. San Juan, ${ }^{1}$ \\ Cecilia L. Llave, ${ }^{1}$ Catherine Banwell ${ }^{4}$ and Erlidia F. Llamas-Clark ${ }^{1}$ \\ ${ }^{1}$ Department of Obstetrics and Gynecology, College of Medicine and Philippine General Hospital, University of the Philippines Manila \\ ${ }^{2}$ Department of Health Region V Philippines \\ ${ }^{3}$ Department of Health Policy and Administration, College of Public Health, University of the Philippines Manila \\ ${ }^{4}$ Research School of Population Health, Australian National University
}

\begin{abstract}
Objectives. To A functioning referral system is critical to the maternal health program, especially in the management of obstetric emergencies. This study explored supply-side barriers affecting the effective implementation of the said service delivery network (SDN) in Legazpi, Albay in the Bicol Region of the Philippines.

Methods. Face-to-face in-depth interviews using semi-structured questionnaires were performed with health care providers involved in the SDN in Albay. Extensive note taking was done by the primary investigator while participants were observed during performance of duties from June - November 2018. Interviews were audiorecorded, transcribed, translated into English, and analyzed thematically along with the observation notes using NVivo. A deductive-dominant approach was utilized for the data content analysis.
\end{abstract}

Results. Referral system barriers identified were cross-cutting across the different components of the health system such as governance, human health resource, service delivery and information systems. The barriers were further classified into individual, organizational and external-related factors in relation to the SDN. Examples of barriers included lack of knowledge of protocols and guidelines, lack of coordination between facilities, poor data management, inadequate capacity building opportunities, and constantly changing political landscape and policies.

Conclusions. The study is the first to explore barriers to effective service delivery network in maternal health in the country. Findings from the study provide significant insight to areas of improvement in the SDN that must be addressed to strengthen local health systems, especially with the country's movement towards Universal Health Care where local health systems play a key role.

Key Words: maternal health, service delivery, referral system barriers

\section{INTRODUCTION}

Paper presented at the Regional Maternal Death Surveillance and Response Workshop, Nov. 21, 2018, La Roca Veranda, Legazpi, Albay, Philippines, and at the $2^{\text {nd }}$ International Public Health Conference 2019, March 19, 2019, Hotel Transit, Kuala Lumpur, Malaysia.

Corresponding author: Maria Stephanie Fay S. Cagayan, MD, PhD Department of Pharmacology and Toxicology

College of Medicine

University of the Philippines Manila

Salcedo Hall 547 Pedro Gil St., Ermita, Manila 1000, Philippines

Email: mscagayan@up.edu.ph
Maternal mortality remains a challenge to healthcare systems both locally and worldwide. Global maternal mortality ratio (MMR) was estimated at 216 per 100,000 live births in 2015, a figure far from the Sustainable Development Goals (SDG) target of less than 70 per 100,000 live births by $2030 .^{1,2}$ Similarly, the Philippines' reported MMR for the same year was high at 114 per 100,000 live births, higher than the estimate for Southeast Asia which was at 110 per 100,000 live births. ${ }^{1,3}$

To respond to the need to reduce maternal mortality and strengthen health systems, the Philippine Department of Health $(\mathrm{DOH})$ introduced the service delivery network 
(SDN) in 2014 as part of its Maternal, Newborn, Child Health and Nutrition (MNCHN) strategy. ${ }^{4}$ Defined as a province- or city-wide network of public and private healthcare facilities that can provide MNCHN services, the SDN was introduced to address the gaps in maternal health referral systems and improve coordination across healthcare levels. ${ }^{5}$ The province serves as the basic unit for planning and implementation of the MNCHN strategy which allows different health care providers, both public and private, within the locality to organize themselves into a well-coordinated service delivery network to meet the varying needs of populations and ensure the continuum of care. Reproductive care services, including basic, comprehensive emergency obstetric and essential newborn care and communication and transportation system supporting this network thus become more available and accessible to all.

There are three levels of care in the MNCHN SDN: (1) Community level service providers; (2) Basic Emergency Obstetrics and Newborn Care (BEmONC) -capable network of facilities and providers; and (3) Comprehensive Emergency Obstetrics and Newborn Care (CEmONC) -capable facility or network of facilities (Figure 1). These three levels of care in MNCHN assist in the provision of different services and functions. ${ }^{5}$

Given that most transfer referrals across the threetiered health delivery system are usually emergency or urgent conditions, it is critical that the referral system is functioning optimally. Studies have proven that the timely identification and safe transfer of patients through the three tiers of the healthcare delivery system decrease the incidence of preventable maternal, neonatal and child mortality. ${ }^{6}$ It is estimated that 73 percent of maternal mortality could be averted if all women received appropriate emergency obstetric care. ${ }^{6}$ The effectiveness of the three-tiered MNCHN system depends significantly on the strength of its referral system. ${ }^{6}$
Part of the process in strengthening systems is to identify its weaknesses in order to address them. Several studies from other countries have looked into the strengths and weaknesses of the maternal health referral system. However, there is limited literature on the barriers hindering the effective implementation of an SDN in the Philippines. Only one study has been found in literature search - a rapid baseline assessment conducted by the United States Agency for International Development (USAID) LuzonHealth project in partnership with the DOH in 2015 to determine the needs and gaps in existing SDN settings. ${ }^{6}$ The baseline assessment involved the 21 partner provinces of LuzonHealth. Results of the assessment indicate that only 20 percent of the project sites claimed to have written referral arrangements and agreements among the service delivery facilities. ${ }^{6}$ An assessment of the maternal health SDN referral mechanism was also done in one of the project sites. ${ }^{6}$ Major findings of the assessment include a lack of clear formal referral agreements or guidelines between referring and receiving facilities, the absence of advance arrangements for urgent/ emergency situations, and the unavailability of specialist services in common referral locations, and low referral acceptance resulting from full capacity. ${ }^{6}$

As part of a larger evaluation study of the local maternal service delivery network in Legazpi, Albay in the Bicol Region of the Philippines, this paper aims to explore the barriers to the effective implementation of the said service delivery network from the perspective of health service providers in the area.

\section{MATERIALS AND METHODS}

\section{Study design and study setting}

The study utilized a qualitative approach to an evaluation study using face to face in-depth interviews (IDIs) and

\section{MNCHN Service Delivery Network}

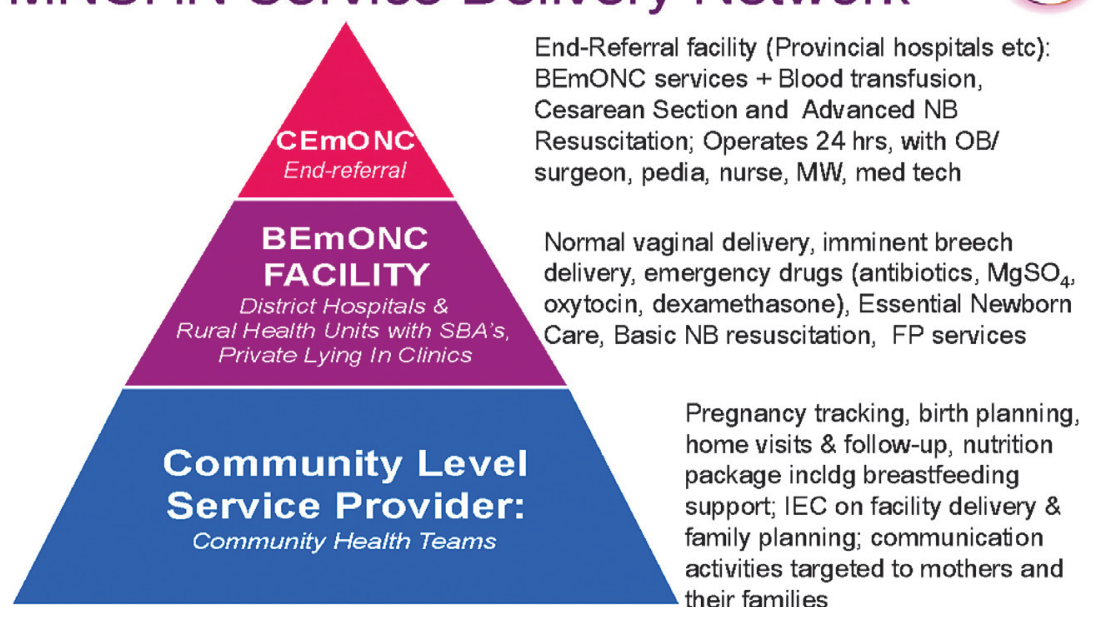

Figure 1. Maternal and Child Health Service Delivery Network. 
participant observation to explore the barriers in the implementation of an effective maternal service delivery network. It was carried out in Legazpi City, Philippines the capital city of the province of Albay, and the largest city in Bicol Region with a population of 196,639 as of the 2015 Philippine Census. ${ }^{7}$ Bicol was chosen as the region of interest because it registered a maternal mortality ratio (MMR) of 123.5 per 100,000 live births in 2015. Legazpi City was then selected as the study site, despite its low reported MMR at 6.1 per 100,000 live births, due to the presence of an operational SDN in the city, and because the largest secondary and tertiary hospitals in the region are located there. ${ }^{8}$ The city also has 4 barangay health centers with birthing stations, 12 private lying-in clinics, and 5 secondary and/or tertiary hospitals. ${ }^{8,9}$

There are three types of facilities that participate in the SDN - rural health units or city health center, public and private lying-in clinics, and hospitals. ${ }^{9}$

\section{Study population}

Participants in the IDIs were health service providers within the Legazpi City SDN as well as provincial and regional health officers who consented to participate in this study. They were purposively recruited for their knowledge, expertise and involvement in the city's maternal health SDN. The primary investigator wanted to get the perspectives of implementers across the different levels of the healthcare system in order to gain an in-depth understanding of the local SDN - how it works and what challenges it faces in implementation. The interview with the regional and provincial health officers provided insights on issues occurring at the macro-level such as policy and guideline development and financing, while those with the clinic staff provided the micro-level perspective with more focus on practical daily operations such as physical transfer of patients from one facility to another and use of referral forms. This mix in type of respondents allowed for the study to have a multi-tiered perspective on SDN implementation surfacing issues of the whole system.

\section{Conceptual Framework}

The study utilized the Integrated Patient-Centered Health Service Framework for Maternal Health developed by the primary investigator as part of a larger study (Figure 2). In addition, the WHO six building blocks of the health system was also used as guide in exploring the input and process indicators within the Integrated PatientCentered Health Service Framework for Maternal Health (Figure 3). The referral mechanism involves communities, primary, secondary and tertiary health units that require coordination and cooperation so that patients are able to access quality care at the right level. Effective referral requires clear and proper communication, adequate resources, appropriate financing and strong governance to ensure that the patient receives optimal care at each level of the system, and facilitates the patient's movement between facilities according to the disease or condition severity. ${ }^{4-6}$

\section{Research Instruments}

IDIs were done using the Referral System Assessment (RSA) Instrument. ${ }^{10}$ The RSA instrument is a semi structured open ended questionnaire designed to collect information from health program managers and service providers in order to evaluate successful implementation of systems. Requiring around 60 to 90 minutes to be completed, the instrument consists of five sections: 1) background characteristics of facility or organization, 2) characteristics of the referral network, 3) referral system monitoring, 4) referral system

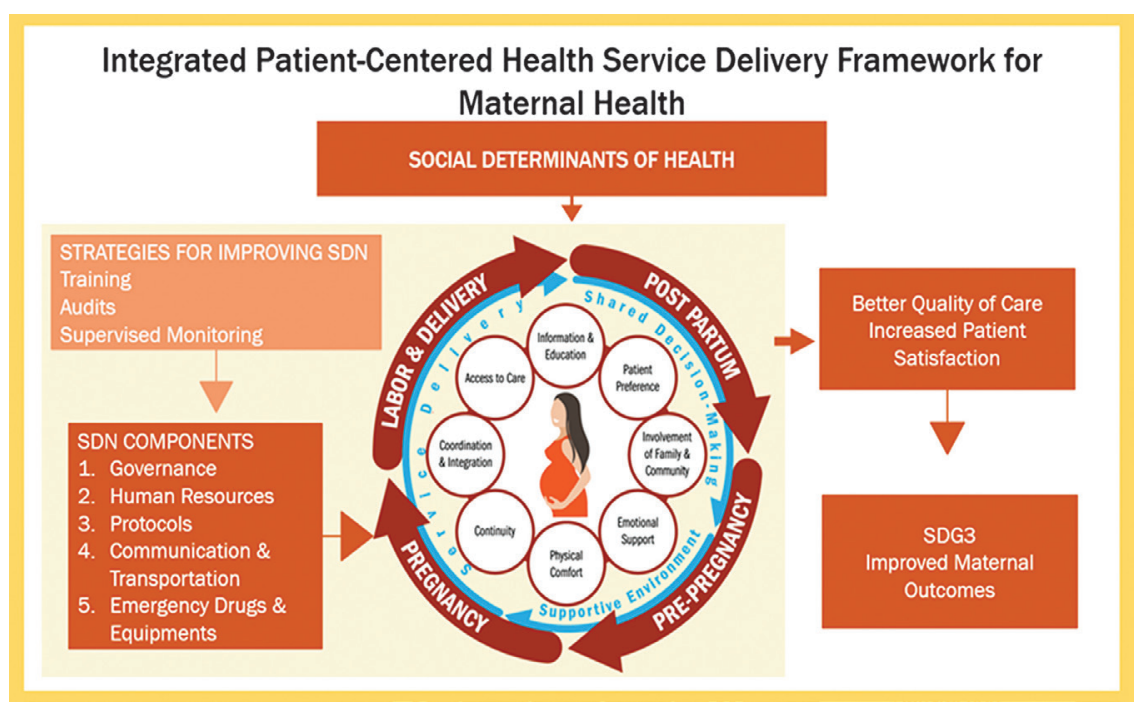

Figure 2. Integrated Patient-Centered Health Service Framework for Maternal Health (Primary Birthing Facilities). 


\section{System building blocks}

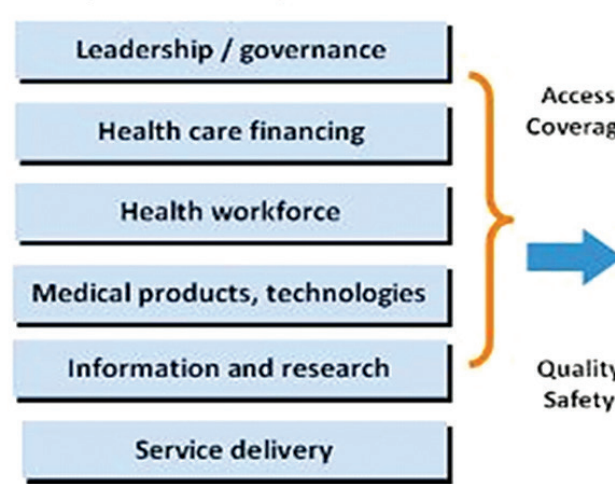

Goals/outcomes

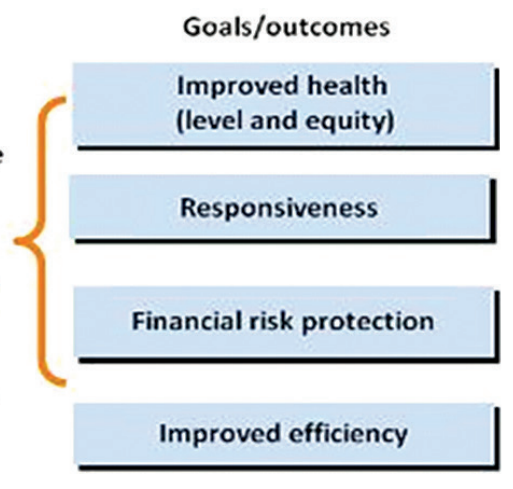

Figure 3. Health system strengthening.

processes such as referral protocols, data quality and use, client confidentiality and satisfaction, and 5) respondent recommendations. Most questions are open-ended to allow respondents to describe the system and its strengths and weaknesses in their own words.

\section{Data Collection}

Data were collected in a span of six months from June to November 2018. IDIs with the health service providers were done by the principal investigator in English and Filipino language using the RSA instrument. The primary investigator scheduled appointments with the participants for the IDIs to avoid disruption of their work schedule and commitments. The interviews were conducted in the health facilities (i.e. lying-in clinics, health center, hospitals and DOH regional office) where the health care providers work. Interviews with the regional and provincial health officers were conducted in the privacy of their own offices, while interviews with clinic staff were done either in private rooms or areas with low foot traffic within the facility to provide as much privacy as possible to the interviewee. However, there were still instances wherein other healthcare staff and some patients were present during the interviews. Interview time took approximately 45-60 minutes to finish.

Direct observation of the workflow, patient flow and dynamics in the area was done by the primary investigator by going on a 6- to 8-hour duty $2-3 x$ in each of the participating facilities within the data collection period. The primary investigator was endorsed by the regional and provincial offices to the participating facilities. Consent from the head of the facilities were also obtained prior to the start of data collection.

The primary investigator documented the healthcare providers' interactions with each other and overall workflow including patient interaction, documentation, reporting and data utilization at the facility, provincial and regional level.

\section{Data Processing}

All interviews were audio-recorded with participants' consent, and transcribed in English by 2 external transcribers who had at least 2 years experience in transcribing field interviews. Two transcribers were utilized for the data processing to ensure accuracy of audio transcription. Both transcribers listened to the same audio recordings and produced transcriptions with a $70 \%$ interjudge reliability. For areas of incongruence, the primary investigator decided which transcription to use based on recall of the actual interview conducted. Coding of the themes from the interview transcripts and observation notes was carried out in NVivo $12 .{ }^{11}$ The primary investigator and one other investigator independently coded all interview transcripts and observation notes. Consensus on identified themes were done through discussion among the investigators of the study.

\section{Data analysis}

A deductive-dominant approach was utilized for the data content analysis. Using the input and process indicators of the Integrated Patient-Centered Health Service Framework for Maternal Health as guide, thematic analysis was done based on identified parts of the health provider's narratives that describe their experiences with the city's SDN and the primary investigator's observation notes. Resulting themes were further categorized into individual, organizational and external level barriers depending on the nature of their occurrence in relation to the SDN.

\section{Ethics}

The study received ethical clearance from the University of the Philippines Manila Research Ethics Board (UPM REB) as part of the bigger evaluation study done by the principal investigator (UPM REB 2018-010-01).

\section{RESULTS}

Seventeen health service providers participated in the IDIs. There were 1 male doctor, 2 male nurses and 1 male non-government organization (NGO) coordinator. The rest were female health workers -1 regional $\mathrm{DOH}$ officer, 2 provincial health officers, 1 medical doctor, 3 nurses, 4 
midwives, and 2 who were practicing as both nurses and midwives. The average years in service was 15 years. Four midwife clinic owners were interviewed from the private sector. A summary of their profile is shown in Table 1.

Resulting themes based on the input and process indicators were classified into individual, organizational and external factors (Table 2).

\section{Individual-level barriers}

Individual-level barriers are defined as factors related to the healthcare worker's individual capacity to provide services across the SDN.

\section{Lack of knowledge of one's roles in the health care team}

Lack of knowledge in performing specific interventions such as emergency medication administration among front liners in the primary care facilities resulted in unnecessary referrals to the tertiary hospitals which causes delays in care provision despite adequate resources in the facility and presence of competent staff.

Table 1. Summary of respondent profile

\begin{tabular}{ll}
\multicolumn{1}{c}{ Profile } & \multicolumn{1}{c}{ Frequency } \\
Sex disaggregation & 4 Males \\
& 13 Females \\
\hline Age in years (mean, range) & $39.9(24.0-60.0)$ \\
\hline Degree/License & 5 Registered Midwives (RM) \\
& 6 Registered Nurses (RN) \\
& 2 with both RM \& RN licenses \\
& 2 Medical Doctor (MD) \\
& 1 with both MD and masters in \\
& public health degree (MPH) \\
\hline Years in service (mean, range) & 15.6 (3.0-37.0) \\
\hline Designation & 12 public health workers \\
& 8 clinic staff \\
& 4 with administrative positions \\
from the hospital, provincial and \\
regional offices \\
4 private midwives \\
1 NGO
\end{tabular}

Table 2. Themes and subthemes (barriers)

\begin{tabular}{ll}
\multicolumn{1}{c}{ Theme } & \multicolumn{1}{c}{ Subthemes (barriers) } \\
Individual & - Lack of knowledge of role in healthcare teams \\
& - unwillingness to learn new skills \\
& - competition between public and private \\
& healthcare workers (unexpected finding) \\
\hline Organizational & - Lack of and poor implementation of \\
& guidelines/protocols \\
& - Inadequate capacity-building opportunities \\
& - Poor coordination between facilities \\
& - Pack of human health resource \\
& reporting, lack of proper database) \\
\hline External & - Changing political climate \\
& - Rapid changes in policies cascaded from the \\
& national office \\
& - Budget limitations \\
\hline
\end{tabular}

"I know how to give $\mathrm{MgSO}_{4}$ [magnesium sulfate] but the hospital is just 15 minutes away so I would rather that they administer the drug for the preec [preeclampsia] patients there. I am not sure if we are allowed to give it." - Public Health Midwife

\section{Unwillingness to learn new skills}

The mean years of service of the study participants was 15.6 years. Only 6 of the 17 participants had less than 10 years of service, of which only 1 had less than 5 years. It was observed that clinic staff with longer years of service have a disinterest for learning new skills and adopting new guidelines that aim to improve efficiency of service delivery and quality of care. Younger clinical staff were eager to ask clinically-related questions and to confirm if what they are practicing are correct and valid.

"We have a co-worker here in the lying-in. She is almost retiring. She insists that she knows how to make the partograph but her last BEmONC training was 15 years ago and when I look at her labor curve, it does not seem to be correct." - Male, Public Health Nurse

\section{Competition between public and private healthcare} workers (unexpected finding)

Another individual-level barrier affecting the referral system is the competition between public and private healthcare practitioners which hindered referrals between the two sectors. Some midwives were also reported to have their own private practice while still being employed by the local government units.

"Our problem is the private midwives. All they are after is to make more income." - Public Health Midwife

"The private midwives give incentives (300500 pesos) to tricycle drivers, too. They also provide food for the patients and their caretakers during the confinement. That is why we have less patients. Public Health Nurse

Administrators were also asked what their observations were regarding interactions between obstetricians and midwives.

"Obstetricians (OB) have refused to sign Memorandum of Agreement (MOA) with private lying-in to regulate midwives' practice. So you will notice that for private lying-in centers they refer to just one OB and one Pedia." - Public Health Administrator

\section{Organizational Barriers}

Organizational barriers are those which are directly related to the operations of the healthcare facilities (i.e. hospitals, lying-in clinics, rural health clinics) involved in the SDN. It encompasses implementation of guidelines, capacity building of staff, availability of health resources, health information systems, and coordination between facilities. 
Lack of and poor implementation of guidelines/ protocols for the SDN

A major barrier in the smooth implementation of the SDN is the lack of comprehensive guidelines for its implementation and monitoring and evaluation (M\&E). Though M\&E meetings are conducted every quarter, discussions were limited to the evaluation of the referral numbers. Facilities with high referral counts were assessed to be implementing the SDN well, while those with low referral counts were tagged as needs improvement. The monitoring discussions did not tackle the root causes of under referral or even those of high referral. Consequently, the meeting did not involve in-depth discussions on improvement of strategies to further improve the SDN. On document review by the primary investigator, however, appropriate indicators are not lacking.

"There are still no specific instructions for the administrative orders. The SDN is just an offshoot of the Interlocal Health Zone (ILHZ)." - Public Health Administrator

"SDN workshops are still works in progress. There are still referrals that are unclassified hence we note them." - NGO

"Monitoring is part of program in paper but is not really being implemented. There is really no $M$ and $E$ in the province. There is really no tool available yet." Public Health Administrator

Moreover, a gap exists in monitoring the performance of private facilities.

"Private lying-in facilities are not included in the recognition that they are following standards. They are not included in those being assessed as centers for excellence." - Public Health Administrator

Further, poor communication of SDN guidelines and protocols hindered the implementation of the SDN at its full capacity as some facilities, especially the private facilities, were not aware of updated guidelines and referral categories. The most common mechanism of information dissemination to facilities was through an electronic mail, often to personal accounts rather than a central facility account which results in reception issues due to high turnover of facility staff or lack of dissemination of the recipient to other staff in the facility. Additionally, the staff sent to attend SDN meetings often fail to feedback the proceedings to the team in the facility.

"Majority do not read soft copy of memo we sent to them regarding referral categories and guidelines." Public Health Administrator

"We know that we have an SDN agreement but I have not seen the document. We refer patients based on our knowledge of what high risk patients are." Private Midwife
Further, not all facilities were aware of the quarterly M\&E meetings, and therefore, were not able to participate. Some who were able to attend were not properly equipped to participate in the discussion as they failed to bring the necessary data needed.

"We are not able to go around regularly. We had one workshop last year, but the staff did not bring the necessary records and data." - Public Health Administrator

\section{Lack of human health resources}

Staffing shortages and fast staff turnover are major barriers in service delivery across the different components of the SDN. Most of the staff in public facilities were only employed under contract as slots for permanent positions were limited. Most importantly, most facilities did not have a permanent SDN point person to handle the program. Often, those assigned to perform functions for the SDN such as coordination across facilities, and data encoding and reporting were burdened with multiple roles outside of their SDN functions which hindered them from doing their SDN tasks properly.

"There is fast turnover of health professionals from one facility to another and from private to government and private to private." - Public Health Administrator

"We have hospitals that have ready facilities and ORs unfortunately with no available doctors. Unfortunately, we have facilities that have been upgraded but the human resources did not catch up." Public Health Administrator

\section{Inadequate capacity-building opportunities}

Capacity-building workshops are conducted by the DOH regional office. Healthcare workers complained of limited opportunities for capacity-building. Given the high staff turnover in most facilities, there are staff, for example, who remain untrained on SDN guidelines and protocols. Further, given the limited slots for training attendance, only one representative is invited in such trainings, who are often the head of the facility rather than the frontliners who are rendering care to the patients.

"One of our problems with regards to training is that there are lack of trainors coming in to conduct $B E m O N C$ and limited slots for the whole of the province and even the region. Trainings are coursed through academic institutions and are regional in nature. So until DOH Regional call for training, health staff only undertake memorandum of undertaking that they will train within that year and they will already be given the license to operate." - Public Health Administrator

"I have seen facilities that have 3 Memorandum of Understanding (MOU) for 3 consecutive years because every time they have new staff and the former staff that had to train has already resigned." - Public Health Administrator 
Private midwives also complained about the inequity in training access between them and public healthcare workers. Often, slots have already been filled up by different public facility representatives even before they get to learn that there is an available training program or workshop.

\section{Poor coordination between facilities}

There is no standard communication protocol between facilities resulting in difficulties in coordinating referrals. Referring facilities have no designated staff to accompany the patients to the receiving facility. Communication equipment are also lacking or in poor condition. Common issues encountered include absence of a telephone in the facility, busy telephone line from the volume of calls, and no designated staff to receive calls.

Further, referring facilities do not receive feedback on whether the patient has successfully arrived at the referral hospital.

"In the delays, there is also delay in referring to hospitals because they send the patients to hospitals thinking that hospitals are capable of managing the cases, however, there are no specialist and only generalists. There are hospitals that are supposed to be CEmONC but they do not have capacity to do CS." - NGO

\section{Lack of available transportation between facilities}

Not all facilities are equipped with vehicles to transport patients during referrals. For those who have ambulances available, the most common issue encountered is the absence of an available driver.

"We share one old ambulance between all the public primary care facilities in the city. In the evenings, it is usually parked at the farthest lying-in center. The private birthing facilities were also sharing the same ambulance with us for the past years. However, now they were asked to seek a memorandum of understanding with a private CEmONC hospital." - Public Health Nurse

\section{Poor data management}

The SDN has a weak data management system. Official forms are not filled out appropriately, and the overall compliance rate of facilities with data reporting is below $50 \%$. The most common reason for incomplete data entries is the failure of staff to fill in entries in the logbook.

"We make the entries on our logbooks as was endorsed to us by previous midwives. Sometimes, we are so busy we write on them at the end of the duty or the next day." - Private Midwife

"We accompany patients to the hospital, but the residents take a long time filling up the form. We cannot wait for them so we end up having the slip with only the signature of the clerk, student or resident on it but no other information written down." - Private Midwife

"Private lying-in clinics are busy completing their PhilHealth documents that they are not able to fill up required completion of reports need by provincial health office. PhilHealth documents are priority by private lying-in because they are income generating." - Public Health Administrator

Further, not all facilities have started to implement use of standardized referral logbooks since they were not fully oriented on referral guidelines by the staff who attended the orientation. Reporting protocols are also not standardized for all facilities.

The SDN also has no formal database. The region only has one person to consolidate all data from the facilities in order to determine the aggregate referral number. However, only the number of referrals is counted. The forms are merely collected, counted and stored.

"No soft copy. All data are sent via hand. Forms are uniform for the government but not for private. There are electronic medical records but only for PhilHealth claims. They do not utilize these for report generation of referrals." - Public Health Administrator

"Information technology (IT) officers are usually just encoders and do secretarial job. They just consolidate reports. And they do not reconcile with other project coordinators because we have different deadlines and cut offs." - Public Health Administrator

\section{External Barriers}

The changing political climate in the province and rapid changes in policies cascaded from the national $\mathrm{DOH}$ office are among the external barriers which the SDN has no direct control or influence over. The local health budget and allotment per health program are dependent on the policies and priorities of the current local chief executive. Program guidelines may be cascaded centrally by the $\mathrm{DOH}$, however, strength of implementation relies on availability of resources allocated at the local level.

"Budget increase went to infrastructure and buman health resource but it is going to the public health. However, maternal deaths are happening at the hospital level." - Public Health Administrator

"Requirements for accreditation and licensing keeps on changing." - Public Health Administrator

\section{DISCUSSION}

With the recent legislation of the Universal Health Care (UHC) Law in the Philippines, healthcare systems are moving towards local integration at the city and provincial level for health service delivery. The minimum components stipulated in the law for integrated local health systems include a primary care provider network with patient records accessible throughout the health system; accurate, sensitive and timely epidemiologic surveillance systems; and proactive and effective health promotion programs or campaigns. ${ }^{12}$ Moving towards UHC requires strengthening local health 
systems making the evaluation of existing healthcare delivery models critical in providing insight on the strengths and weaknesses of the health system.

Using the Integrated Patient-Centered Health Service Framework, this is the first qualitative study to assess barriers affecting the effective implementation of the MNCHN SDN in Legazpi, Albay. Supply-side barriers identified in the study were like findings of other studies on maternal referral systems and health referral systems in general. ${ }^{13-15}$ Findings are categorized into individual, organizational and externalrelated factors.

Individual-related barriers consisted of lack of knowledge of one's roles in the healthcare team, unwillingness to learn new skills by those with long duration of service, and the competition between public and private healthcare workers. The first two barriers resulted in unnecessary referrals outside the primary facilities which delayed patient access to care. The competition between public and private health workers, meanwhile, resulted in poor coordination between the two sectors. Though occurring at the individual-level, the health system may still have a hand in addressing these barriers. For example, literature shows that lack of training, skills in networking, epidemiology, research appraisal or critical thinking about clinical practice could limit health workers' awareness and receptivity to clinical practice changes. ${ }^{16}$ Thus, ensuring availability of capacity-building opportunities focused on strengthening these skills may aid in improving the willingness and ability of health workers to deliver care according to evidence-based guidelines.

Organizational-related barriers consisted of issues within the health system to which the SDN has a direct control over. Lack of and improper implementation of guidelines are major barriers to obstetric care access in health facilities. ${ }^{13}$ Similarly, staffing shortages and high staff turnover pose barriers to access to and quality of care. ${ }^{13,16-21}$ Due to staffing shortages, healthcare workers are burdened with multiple roles and increased workloads which compromises quality of care rendered to clients. ${ }^{16}$ Further, retention of healthcare workers is a major challenge in the Philippine healthcare system given that in order to compensate for the absence of permanent positions, a large proportion of service providers are hired on temporary contracts. This culture of temporary work contracts proves problematic for effective health referral systems as literature have shown that they serve as barriers to care coordination due to the resulting staff rotation which produces loss of qualified human resources. ${ }^{17}$ Literature has also shown that recruitment of contractual staff, though convenient and economical for the government, does not result in any substantial improvement in the utilization of maternal health services. ${ }^{19}$ Lack of professional training also serves as a major obstacle in implementing care coordination. It results in failure to adequately exercise responsibilities and unwillingness to collaborate due to lack of awareness of guidelines. ${ }^{16,17}$ Capacity-building issues encountered in the Legazpi SDN were rooted in financial and human resource limitations which would require longterm solutions.

Poor coordination and feedback mechanisms between facilities reduces the effectiveness of referral systems. ${ }^{22,23}$ The absence of a pre-referral and feedback mechanism between referring and receiving facilities is the primary reason for coordination issues in the referral network. ${ }^{14}$ Ideally, the referring facility must provide adequate patient information to the receiving facility. Likewise, receiving facilities should record the outcome of visit and treatment of all referred patients in a standard form, and should report the followup recommendations to the referring center. ${ }^{23}$ Insufficient information transferred from the primary care providers to specialists in the referral hospital serve as a hindering factor in providing continuing care to referred patients. ${ }^{17}$

Apart from proper coordination and communication, the transportation of clients between facilities is a critical step in a successful referral. Consequently, the availability of a reliable emergency transport system plays an important role in the timely delivery of maternal health services as it enables the timely transfer of patients between health facilities and facilitates easy referral between the different levels of care. ${ }^{24}$ Thus, the SDN must take measures to ensure that access to a reliable emergency transport system is always available to its clients.

Data play an important role in the delivery of healthcare services. With an efficient and accurate data management system, organizations are able to consolidate, centralize, access and ultimately, measure and analyze health indicators to monitor and evaluate effectivity of implemented programs. Development of evidence-based strategies and policies to improve healthcare delivery would not be possible without data. ${ }^{25}$ Access to both clinical and even claims data helps policy makers uncover strategies which will be helpful in analyzing health workers attitudes, behaviors and loyalties. These initiatives help organizations achieve successful physician alignment, increase volumes and referral rates, and realize incremental revenues. However, the existence of issues in data quality result in underutilization of data for patient care improvement. Other studies have also noted incomplete accomplishment of healthcare forms as a barrier to quality care provision. ${ }^{14,25}$ Measures must be taken to improve the SDN information system to ensure timely, complete and accurate reporting from different facilities. The establishment of a computerized referral information system, for example, would facilitate faster data sharing between facilities for care management, and ensure access of program coordinators to regularly updated data for monitoring and evaluation.

In a decentralized healthcare system such as the Philippines, management of health programs pose administrative and political challenges. Health policies are developed at the national DOH office and cascaded to regional, provincial and city offices. Most healthcare logistics are procured and disseminated the same way. However, budget allocation for health program implementation and 
human resource complement are highly reliant on local government funding and sometimes, external sources of funding. Given this, the success of local health programs depends on investment and support of policy makers and chief executives at the local level which are outside the health program's direct control. The challenge now is to advocate for local support and investment to these policymakers to ensure effective implementation of the health program.

In Legazpi City, solutions to address these barriers are underway. The presence of provincial and local policies, allocation of funds as well as an organized Interlocal Health Zone SDN board that meets regularly is indicative of efforts directed at improving service delivery. This may be partly due to the strong presence of an NGO, USAID's LuzonHealth, that actively coordinates, monitors and evaluates the different activities related to MNCHN SDN. Albay, along with 20 other partner provinces, was chosen by USAID's LuzonHealth project in 2015 for a baseline needs and gap assessment in existing SDN settings. ${ }^{6}$ This project was in support of the DOH's thrust, through its regional offices, to build on gains of earlier reform policies via the Philippine Health Agenda for UHC.

\section{CONCLUSION}

Though the Legazpi SDN has grown since its establishment in 2016, there is much to be improved to ensure quality care provision, and to reduce maternal mortality. A pioneer study to explore barriers in maternal health SDN in the Philippines, findings of this research provide significant insight on areas to improve on in the SDN. Health systemrelated barriers in the SDN warrant a multisectoral approach that takes into consideration the complex and multifaceted nature of the network. Solutions could not come from one actor alone. Every component of the SDN from the DOH regional office to the lying-in clinics must take an active role to address the identified barriers in the network.

As the Philippines move towards Universal Health Care, local health system integration will become the center point of healthcare delivery. The SDN serves as an early model of the city-and provincial-wide health system integration which the UHC Law hopes to achieve. Given this, prioritization of the SDN in the agenda of regional, provincial and city health leaders will be a significant step towards health system strengthening in preparation for the UHC implementation. Significant investments on human health resource and transportation services, a standardized interfacility communication mechanism, and a computerized referral information system may result in improved effectiveness of the SDN leading towards the successful reduction of maternal mortality in the province.

\section{Acknowledgments}

We would like to thank the healthcare providers that allowed this study to be conducted successfully. We would also like to express our sincerest gratitude to the editors and referees for their valuable inputs and comments to this manuscript.

\section{Data Availability}

The qualitative data used to support the findings of this study are available from the corresponding author upon reasonable request to protect the confidentiality of the respondents.

\section{Statement of Authorship}

All authors participated in research conceptualization and data interpretation and analysis, critical revision of article and final approval of version to be published.

\section{Author Disclosure}

All authors declared no conflicts of interest.

\section{Funding Source}

This paper was funded by a grant from the National Institutes of Health, University of the Philippines Manila [Project Code: NIH 2018-001].

\section{REFERENCES}

1. World Health Organization. Trends in maternal mortality: 1990 to 2015: Estimates by WHO, UNICEF, UNFPA, World Bank Group and the United Nations Population Division [Internet]. 2015 [cited 2019 May 30]. Available from: https://www.who.int/reproductive health/publications/monitoring/maternal-mortality-2015/en/.

2. World Health Organization. SDG 3: Ensure healthy lives and promote well-being for all at all ages [Internet]. [cited 2019 May 30]. Available from: https://www.who.int/ sdg/targets/en/.

3. Maternal mortality in 1990-2015 - Philippines.

4. Department of Health (Philippines). Department of Health MNCHN Strategy Manual of Operations [Internet]. 2011 [cited 2019 Jul 15]. Available from: https:/www.doh.gov.ph/sites/default/ files/publications/ MNCHNMOPMay4withECJ.pdf.

5. Department of Health (Philippines). Guidelines in Establishing Service Delivery Network [Internet]. [cited 2019 Jul 15]. Available from: https://www.doh.gov.ph/sites/default/files/publications/ Guidelines\%20 EstablishingSDN.pdf.

6. Luzon Health - Integrated Maternal, Neonatal, Child Health and Nutrition/Family Planning in the Philippines [Internet]. 2016 July 29 [cited 2019 Jul 15]. Available from: https:/www.rti.org/impact/ luzonhealth-maternal-neonatal-child-health-nutrition-familyplanning.

7. Philippine Statistics Authority. 2015 Philippine Census - Bicol Region [Internet]. 2015 [cited 2019 Jul 15]. Available from: https://www. psa.gov.ph/sites/default/files/attachments/hsd/pressrelease/R05.xlsx.

8. Department of Health (Philippines) - Regional Office V. 2015. Bicol Health Profile. Estevez V. 2018 July. Albay Service Delivery Network (SDN) for FP/MNCHN Services. Legazpi City, Philippines.

9. Department of Health - Regional Office V. (n.d.). FP-MNCHN Referral Guidelines for LEDACAMARA (Legazpi, Daraga, Camalig, Manito, Rapu-rapu) Service Delivery Network.

10. MEASURE Evaluation. Referral Systems Assessment and Monitoring Toolkit [Internet]. 2013 [cited 2019 Jul 15]. Available from: https://www.measureevaluation.org/resources/publications/ms13-60?fbclid=IwAR2KRR-jfvN1E1r2TjqstDk0Hh5ebMPQyWvTG Uq3cabMekuD88xiNZoomeM.

11. NVivo 12 (Version 12). Melbourne, Australia: QSR International. 2018 . 
12. 17th Congress of the Philippines. Universal Health Care Act, Pub. L. No. 11223. 2018.

13. Kyei-Nimakoh M, Carolan-Olah M, McCann TV. Access barriers to obstetric care at health facilities in sub-Saharan Africa - a systematic review. Syst Rev. 2017 Jun; 6(1):110.

14. Afari H, Hirschhorn LR, Michaelis A, Barker P, Sodzi-Tettey S. Quality improvement in emergency obstetric referrals: qualitative study of provider perspectives in Assin North district, Ghana. BMJ Open. 2014 May; 4(5):e005052.

15. Pembe AB, Carlstedt A, Urassa DP, Lindmark G, Nyström L, Darj E. Effectiveness of maternal referral system in a rural setting: a case study from Rufiji district, Tanzania. BMC Health Serv Res. 2010 Dec; $10: 326$.

16. Munabi-Babigumira S, Glenton C, Lewin S, Fretheim A, Nabudere $\mathrm{H}$. Factors that influence the provision of intrapartum and postnatal care by skilled birth attendants in low- and middle-income countries: a qualitative evidence synthesis. Cochrane Database Syst Rev. 2017 Nov; 11(11):CD011558.

17. Vargas I, Mogollón-Pérez AS, De Paepe P, Ferreira da Silva MR, Unger JP, Vázquez ML. Barriers to healthcare coordination in market-based and decentralized public health systems: a qualitative study in healthcare networks of Colombia and Brazil. Health Policy Plan. 2016 Jul; 31(6):736-48.

18. Nair M, Yoshida S, Lambrechts T, Boschi-Pinto C, Bose K, Mason EM, et al. Facilitators and barriers to quality of care in maternal, newborn and child health: a global situational analysis through metareview. BMJ Open. 2014 May; 4(5):e004749.
19. Singh A. Supply-side barriers to maternal health care utilization at health sub-centers in India. PeerJ. 2016; 4:e2675. doi: 10.7717/ peerj. 2675 .

20. Onta S, Choulagai B, Shrestha B, Subedi N, Bhandari GP, Krettek A. Perceptions of users and providers on barriers to utilizing skilled birth care in mid- and far-western Nepal: a qualitative study. Glob Health Action. 2014 Aug; 7:24580. doi: 10.3402/gha.v7.24580.

21. Sharan M, Ahmed S, Naimoli JF, Ghebrehiwet M, Rogo K. The World Bank Group [Internet]. 2010. [cited 2019 Jul 15]. Available from: https://www.rbfhealth.org/sites/rbf/files/eritrea\%20maternal $\% 20$ paper.pdf.

22. Give C, Ndima S, Steege R, Ormel H, McCollum R, Theobald S, et al. Strengthening referral systems in community health programs: a qualitative study in two rural districts of Maputo Province, Mozambique. BMC Health Serv Res. 2019 Apr; 19(1):263.

23. Eskandari M, Abbaszadeh A, Borhani F. Barriers of referral system to health care provision in rural societies in Iran. J Caring Sci. 2013 Aug; 2(3):229-36.

24. Kananura RM, Kiwanuka SN, Ekirapa-Kiracho E, Waiswa P. Persisting demand and supply gap for maternal and newborn care in eastern Uganda: a mixed-method cross- sectional study. Reprod Health. 2017 Oct; 14(1):136.

25. Raheja K, Dubey A, Chawda R. Data analysis and its importance in health care. Int J Comput Trends Technol. 2017 Jun; 48(4):176-80. 